\title{
The role of dedicated instrumentation in total hip arthroplasty
}

\author{
Antonio Capone $\cdot$ Daniele Podda $\cdot$ Roberto Civinini $\cdot$ \\ Marco Italo Gusso
}

Received: 10 February 2007/ Accepted: 12 October 2007/Published online: 22 May 2008

(C) Springer-Verlag 2008

\begin{abstract}
Tissue-sparing surgery is a surgical strategy aimed to reduce tissue damage in joint replacement. This can be achieved by reducing soft tissue trauma, performing minimally invasive access routes and limiting bone removal with implantation of conservative prostheses. In order to facilitate mini-approaches, special instrumentation was developed to avoid impingement of the soft tissues and provide an easier and more correct placement of the components. We performed an analysis of the literature and a research of the instrumentation available today, to evaluate the actual utility of dedicated tools.
\end{abstract}

Keywords Conservative prosthesis - Hip arthroplasty · Instrumentation · Minimally invasive surgery

\section{Introduction}

Tissue-sparing surgery (TSS) is a "philosophy" that synthesizes new strategies in joint replacement surgery [1]. The main features of TSS are: perform surgical approaches that respect the anatomical structures causing little trauma to soft tissues, reduction of perioperative blood loss, implantation of conservative prosthesis that allows sparing of bone stock, restoration of physiological joint

\footnotetext{
A. Capone - D. Podda

Orthopaedic Department, University of Cagliari, Cagliari, Italy

R. Civinini · M. I. Gusso

II Orthopaedic Clinic, University of Florence, Florence, Italy

A. Capone $(\bowtie)$

Clinica Ortopedica e Traumatologica, Ospedale Marino,

Lungomare Poetto, 09126 Cagliari, Italy

e-mail: anto.capone@tiscali.it
}

biomechanics, and the use of dedicated instrumentation that can be completed with the aid of computed navigation systems.

While minimally invasive surgical approaches have been described and widely employed, several derive from conventional approaches to the hip joint and others have been specifically developed for minimally invasive surgery.

Minimally invasive approaches use anatomical intervals avoiding the dissection of tendons and muscles as much as possible. The other important aspect of tissue sparing is the reduced sacrifice of the bone stock using implants that require little bone resection. New concepts of TSS are fused into a new prosthesis design of femoral stems and acetabular shells. These satisfy the exigencies of sparing bone tissue and implants that could be easily implanted with minimally invasive techniques. Optimization of the surgical procedure derives by the cooperation between surgeons and manufacturers that developed dedicated instrumentation for surgical exposure and component positioning. The debate in the orthopedic community regarding the real need of this special instrumentation is still on. We performed an analysis of the literature combined with a research on the availability of special instrumentation for minimally invasive surgery provided by the companies, to evaluate the proper role of the dedicated instruments in the tissue-sparing total hip arthroplasty.

\section{Dedicated instrumentation}

The dedicated instruments for minimally invasive techniques are used to improve the surgical exposure of the joint in conditions of limited exposure influenced by the 
dimensions of the wound. The main difficulties in this type of surgery are the poor visualization of the anatomic structures and the troublesome preparation and positioning of prosthetic components [2].

A dedicated cut guide for the femoral neck is available on most of the instrumentation to allow the surgeon to resect at the desired level, the femoral neck, using the greater trochanter as a landmark, without extending the exposure to the lesser trochanter (Fig. 1).

Hohmann retractors with long handles and various angulations have been developed so that the assistant's hands are distanced from the operative field. Flanged retractors have been developed to reduce the number of instruments in the wound. The flanges allow retraction of the surrounding soft tissue when the handle is rotated about the axis of the retractor point.

In the postero-lateral approach, an angled flanged retractor is placed on the anterior wall of the acetabulum to retract the proximal femur anteriorly and to hold back the anterior-inferior capsule with the flanged portion pointing caudal (Fig. 2). A single point retractor is placed under the transverse acetabular ligament to provide inferior acetabular exposure. In the lateral mini-approach, a double point retractor is placed on posterior acetabular wall retracting both the capsule and the femur posteriorly, while a curved single point retractor is positioned on the anterior wall to hold the glutei muscles (Fig. 3). The retractors have light holders and can be fitted with a fiber optic light source that shines directly into the wound.

The reamers for the preparation of the acetabulum have also been modified. In offset reamer an angled handle is provided to avoid impingement of the soft tissues and avoid eccentric reaming (Fig. 4).

The geometry of the reamers has been changed to a lowprofile and to a non-hemispherical shape for easier access

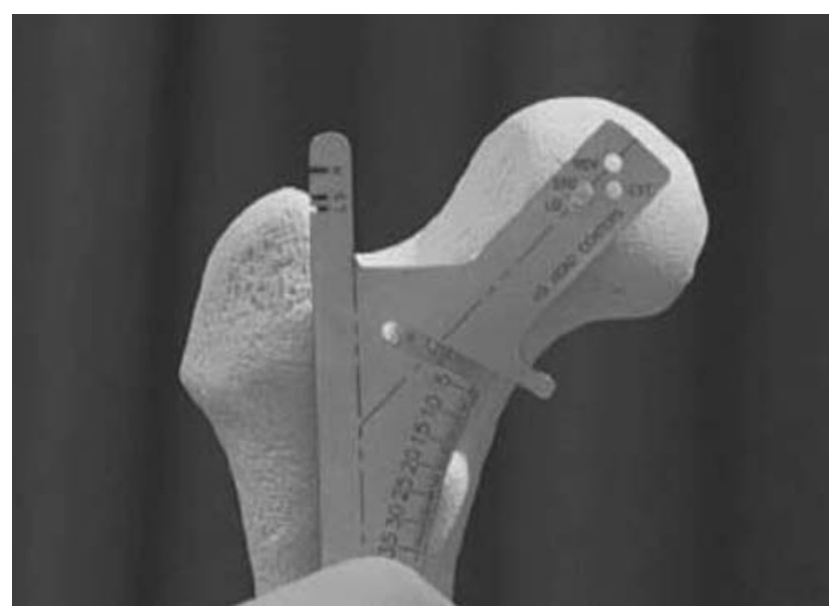

Fig. 1 The resection guide allows to cut the femoral neck using the great trochanter as a landmark

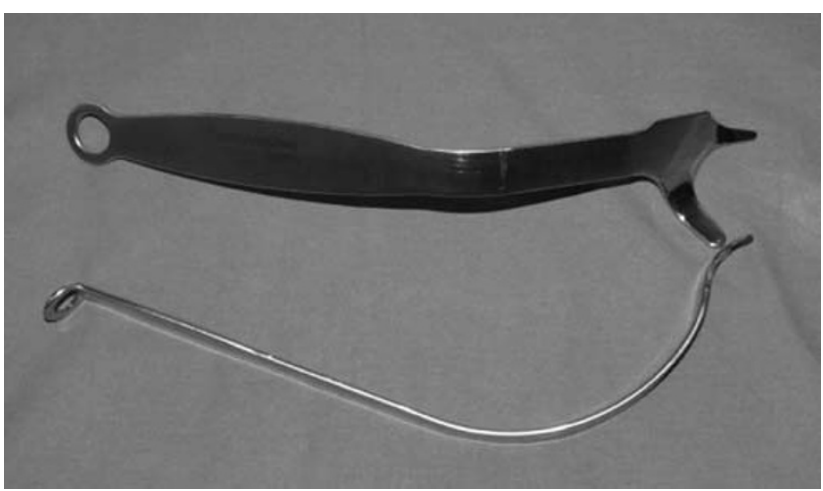

Fig. 2 Angled flanged retractors

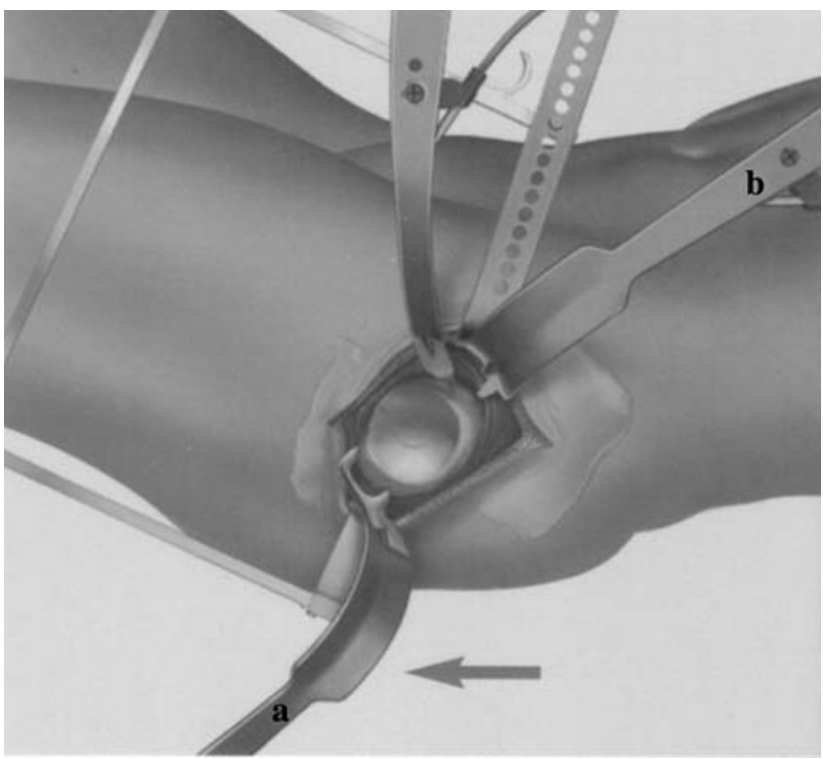

Fig. 3 Positioning of modified Hohmann retractors in lateral approach; offset double point retractor (a) and single point retractor (b) (Zimmer, Inc., Warsaw, IN, USA)

to the wound (Fig. 5). The impactor for the acetabular shell can be angled to reduce the impingement of the soft tissues in positioning the definitive acetabular cup avoiding a vertical shell position.

The impactor for the shell can be provided with lateral alignment frame that gives an exact $45^{\circ}$ abduction and $20^{\circ}$ forward flexion of the shell (Fig. 6).

A modified femoral elevator is available for femoral preparation. The contoured femoral elevator holds the proximal femur out of the wound and protects the proximal pole of incision. In the lateral approach, it is placed on the lateral aspect of the great trochanter while an angled Hohmann is placed medially to the femoral neck (Fig. 7). In the postero-lateral approach the modified femoral elevator is generally positioned over the anterior border of the femur. 

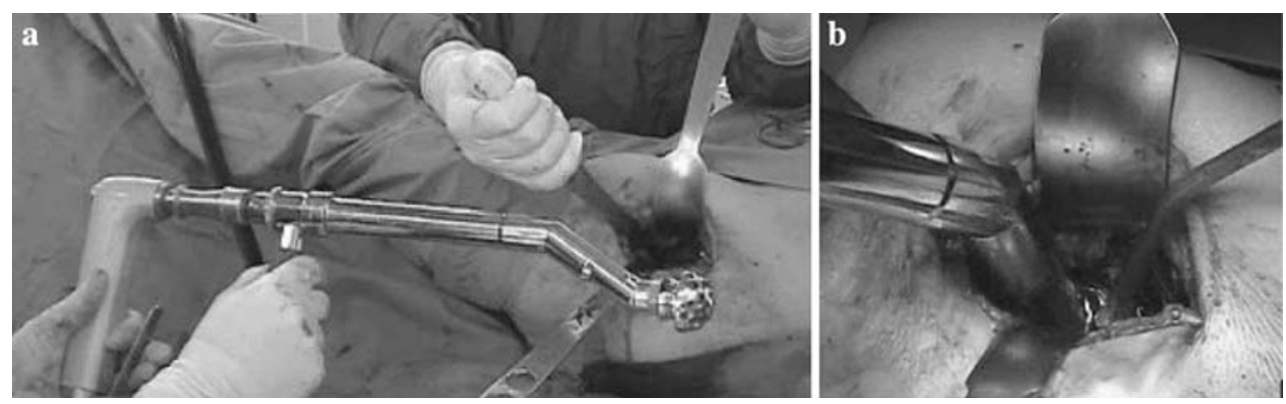

Fig. 4 Offset reamer reduces impingement of soft tissues
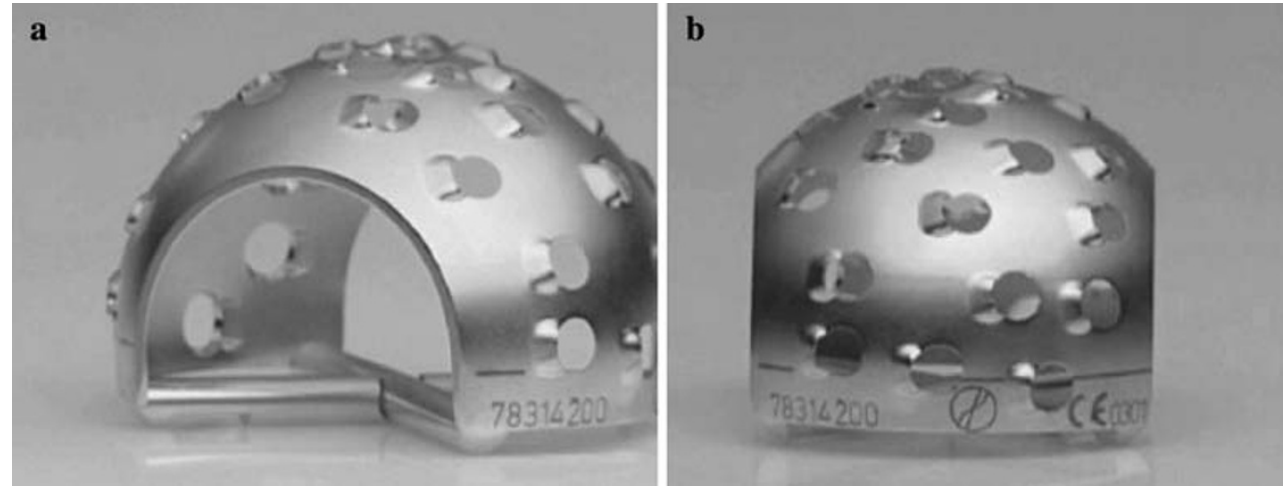

Fig. 5 Modified low-profile acetabular reamer

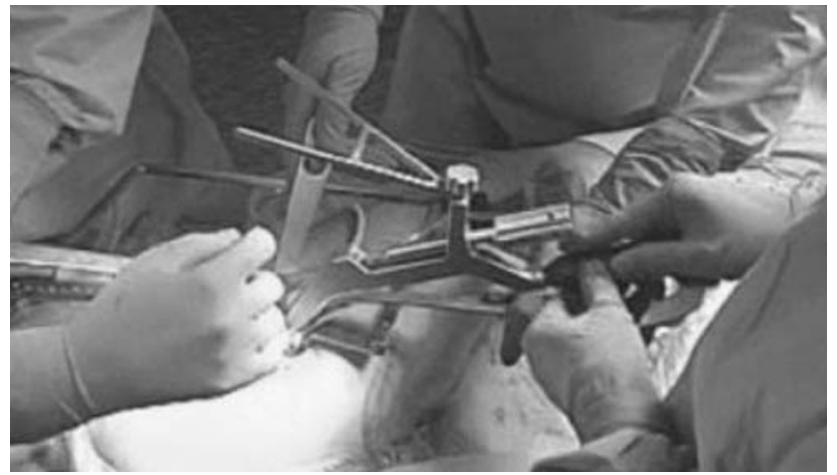

Fig. 6 Offset inserter with alignment frame provides better orientation of acetabular component
The conventional instrumentation for femoral preparation has been modified in the sense of reducing impingement on the soft tissues, so the broach handles are thinner and some companies have even provided curved handles. The choice of the broach handle, either straight or angled, depends on the surgical approach; in anterior or lateral approach the femoral preparation generally is easier with curved broach handles (Fig. 8).

This problem is generally avoided during the insertion of a neck preservation stem because their entrance point is more medial and the curved design of broach is well adapted to mini-approach.

Trial head and neck components have been modified by presenting a lateral slot that allows lateral insertion of both neck and head trials in little space.
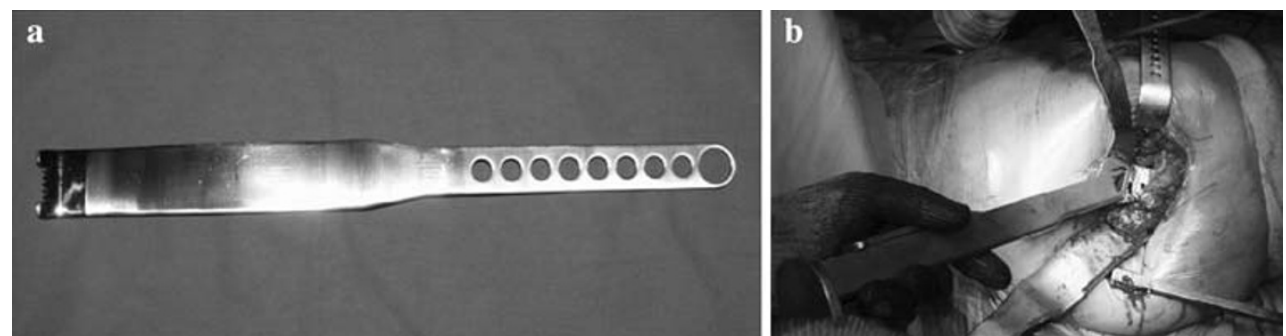

Fig. 7 In the lateral approach the femoral elevator is placed on the lateral aspect of the greater trochanter 


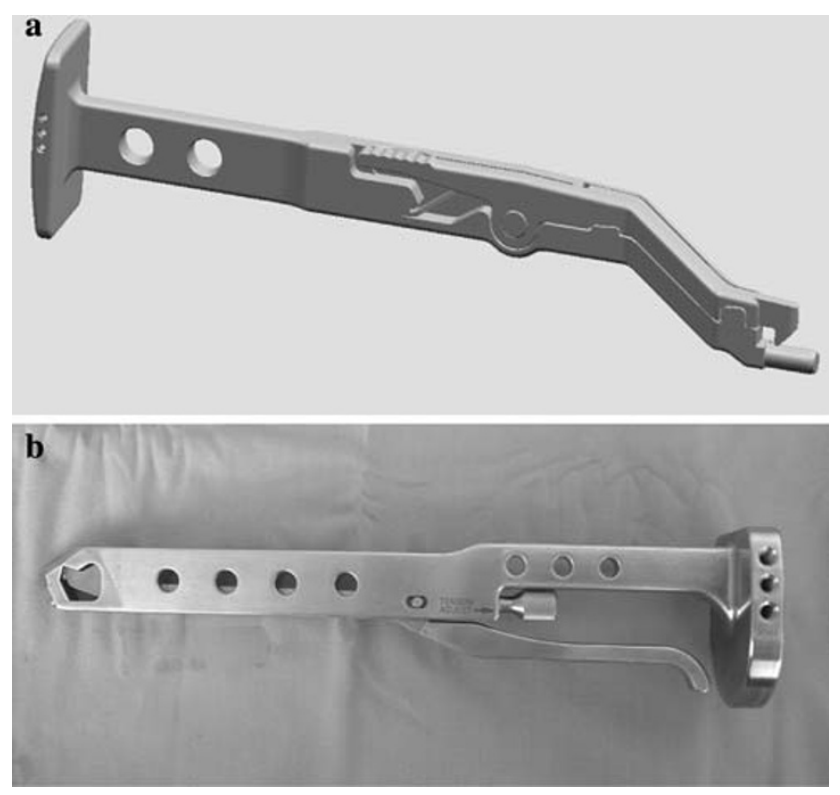

Fig. 8 Angled (a) and straight (b) thin broach handle

\section{Bone stock sparing implants}

Application of TSS concepts guides the development of new femoral stems and acetabular shells.

Models of femoral stems that preserve bone tissue are short and have a proximal load to the femur [3]. The short stem transfers mechanical loads to the proximal femur reproducing physiological load distribution to both the medial and lateral cortex of the bone segment. Stems with retention of the femoral neck transfer loads to the proximal femur along the axis of the neck, reproducing physiological orientation of the trabecular bone. Short and femoral neck preserving stems have the advantage of easily being implanted with a minimally invasive approach. They require a femoral approach without exposing the greater trochanter and without sacrificing the abductor insertion. The shape of the prosthesis and of the instrumentation has a more anatomical design and can be employed without wide dissections, differing from classic straight stems (Fig. 9).

Acetabular shells have been produced in order to reduce bone sacrifice (low-profile shell) and to integrate into the stress lines of the hip joint. New tribology solutions like metal-on-metal and ceramic-on-ceramic couplings for hip arthroplasty reduce debris production with higher diameter heads and consequently improve the mechanical and functional properties of the implants. The large diameter heads increase range of motion and reduce the risk of hip dislocation (Fig. 10).

\section{Discussion}

The advent of TSS changes the prospective of minimally invasive surgery and the efforts for developing mini-
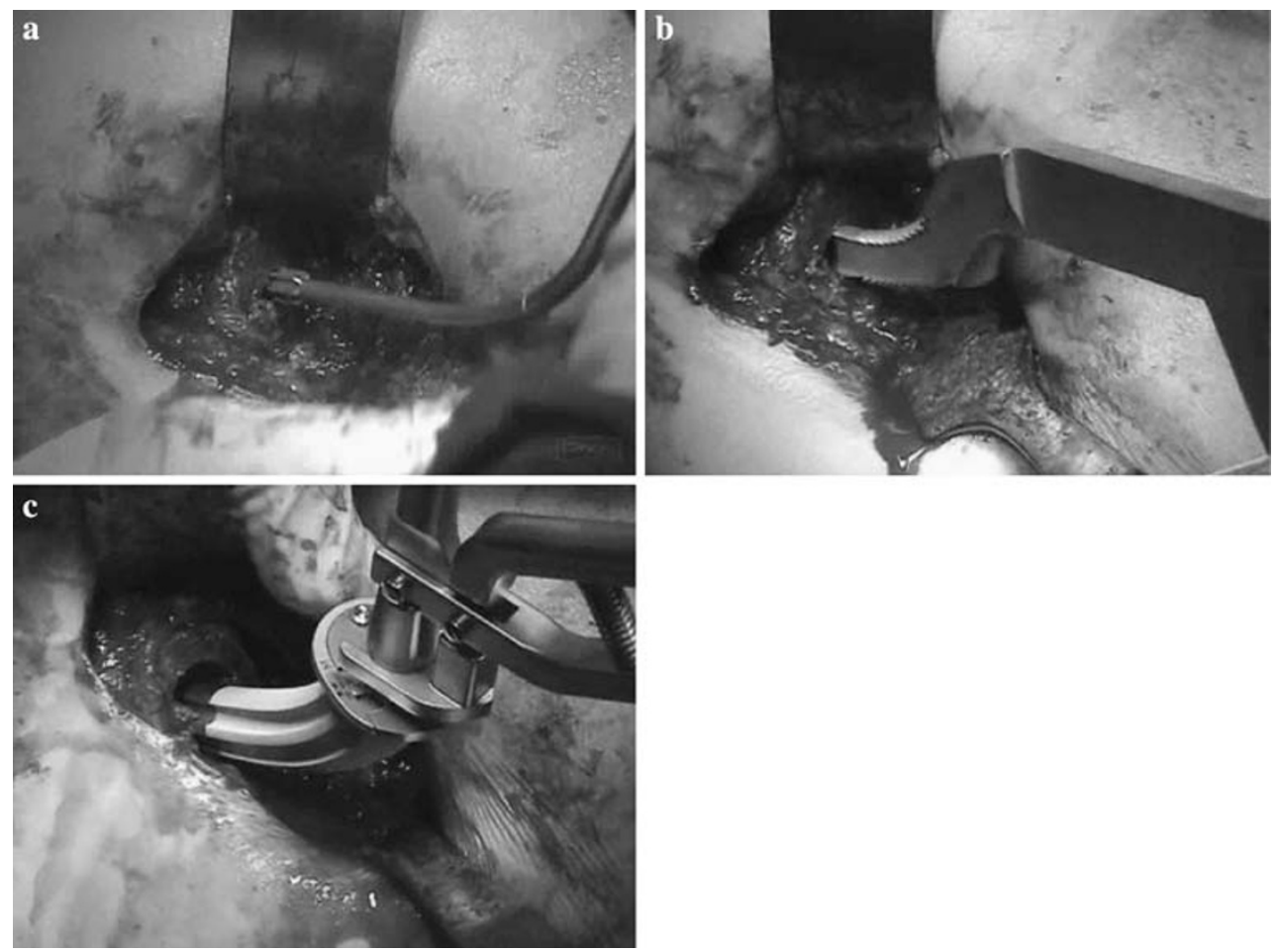

Fig. 9 In the conservative stem medial entry point and anatomical design of instruments reduce impingement of the soft tissues 

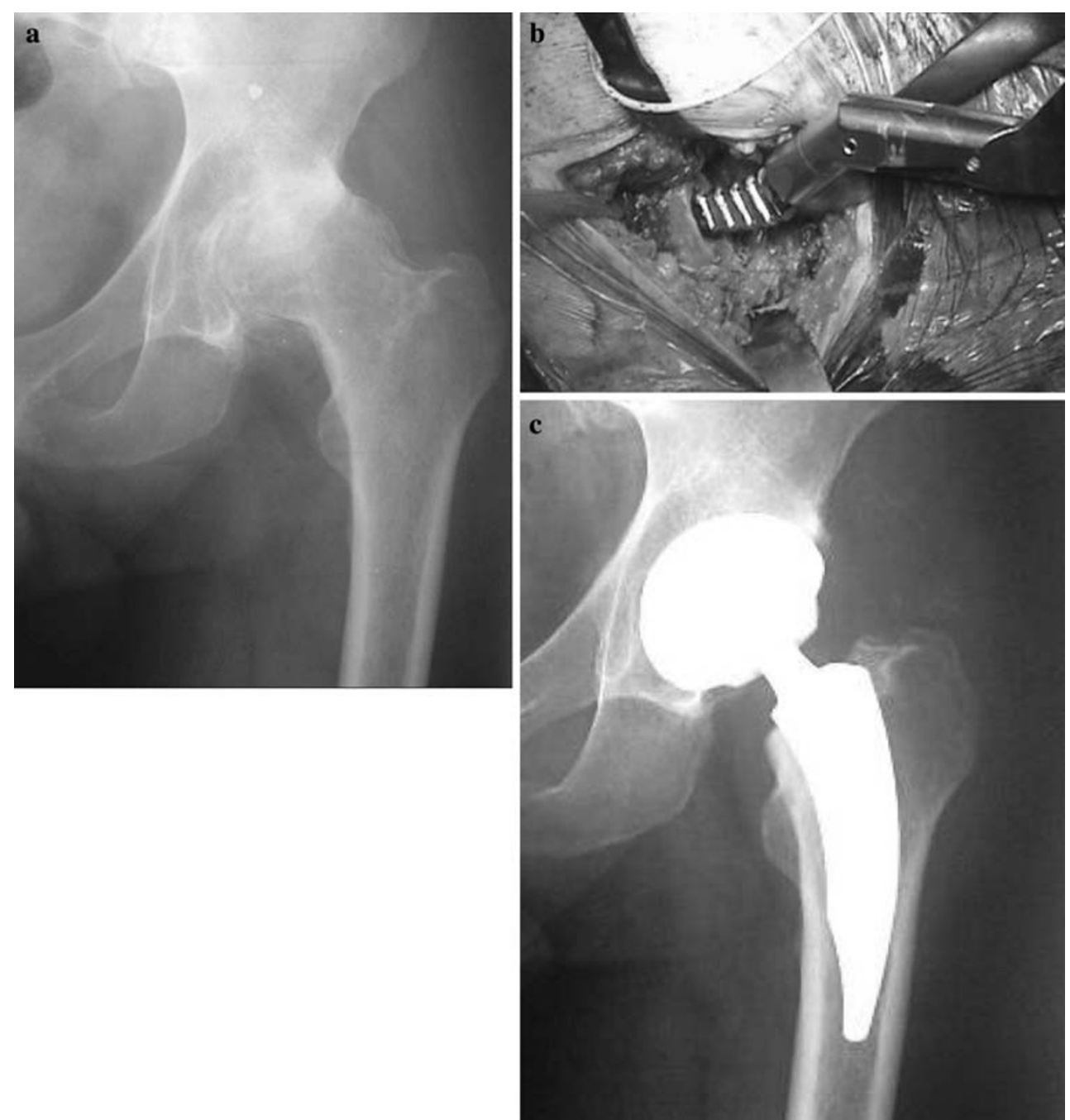

Fig. 10 Preoperative (a) and follow-up radiographs (c) of this total hip arthroplasty synthesize the philosophy of tissue sparing surgery. The implantation through a minimally invasive approach

incisions are now shifted to minimally invasive techniques. The attention of the orthopaedic community is focused not only on the size of the skin incision but also on the surgical damage of muscles, tendons and bone. The difference between mini-incision and minimally invasive approach is also linked with the employment of dedicated implant design and dedicated instrumentation.

Mini-incision techniques have been developed with a principal aim to reduce visible scars, blood loss, and to obtain an earlier discharge of the patient.

In the first series of patients operated with two-incision mini-approach without any preservation of the bone tissue, standard prosthesis with aggressive or cemented stems were used to achieve stability.

Some emerging problems related to the minimally invasive total hip arthroplasty are evidenced by the analysis of the literature. The trouble is mainly linked to the poor visualization of the main anatomical structures and of (b) of a short stem and a coupling ceramic on ceramic with a large diameter head, respects to the anatomical structures and restores joint biomechanics

the landmarks that are usually exposed by a standard approach.

The most frequent minimally invasive surgical approach described in the literature is the mini-postero-lateral incision with sparing of quadratus femoris insertion. Hartzband recommended specific surgical training and dedicated instrumentation. In his series of 100 MIS hip arthroplasties, he reported very few complications. The most important was deep venous thrombosis (four cases) without infection or dislocation [4].

The lateral approach is less frequently used. Berger described a modified lateral approach with only $25 \%$ of the abductors taken off the trochanter. The gluteus medius is longitudinally divided at the junction between the anterior quarter and posterior three quarters of the muscle. An L-shaped incision in the gluteus minimus tendon is then made, the incision begins proximally along its fibers and distally curved laterally to exit inline with the incision in 
Table 1 The incidence of complication in comparative studies between standard and minimally invasive total hip arthroplasty

\begin{tabular}{lllll}
\hline Authors & $\begin{array}{l}\text { Surgical } \\
\text { approach }\end{array}$ & $\begin{array}{l}\text { Dedicated } \\
\text { instrumentation }\end{array}$ & $\begin{array}{l}\text { Early complications } \\
\text { in MIS (\%) }\end{array}$ & $P$ \\
\hline Woolson [8] & Post-lat & No & 12 & 0.02 \\
Hartzband [4] & Post-lat & Yes & 0 & NS \\
Dorr [11] & Post-lat & Yes & 2.7 & NS \\
Ogonda [7] & Post-lat & No & 1.8 & NS \\
Chimento [10] & Post-lat & Yes & 1.4 & NS \\
Berger [5] & Lateral & Yes & 1 & NS \\
O'Brien [9] & Lateral & No & 6 & 0.02 \\
Höwell [2] & Lateral & Yes & 4 & NS \\
\hline
\end{tabular}

the gluteus medius muscle. The anterior portions of the medius and minimus glutei are taken off the trochanter in one continuous sleeve distally to the vastus ridge. In his series of 100 minimally invasive arthroplasty with the use of dedicated instruments, he reported the same complications of the standard approach group (one periprosthethic fracture of femur in each group) without infection or dislocation [5].

Clinical results reported by Pipino showed 91 and $6 \%$ of excellent and good, respectively, at 6 year follow-up in his series of 393 total hip replacements with conservative neck prosthesis performed through modified minimal lateral approach that separates the anterior quarter of the gluteus medius and gluteus minimus from the posterior three quarters [6].

Comparative studies between the standard approach and minimally invasive total hip arthroplasty reported a higher early complication rate in the series treated with minimally invasive arthroplasty performed with no dedicated instrumentation (Table 1). Woolson, Ogonda, and O'Brien reported $12,1.8$, and $6 \%$, respectively, of early complications in their series of total hip arthroplasty performed through mini-approach but using standard instrumentation [7-9]. On the other hand, the authors that used dedicated instrumentation reported lower complication rates $(0-4 \%)$ $[2,4,5,10,11]$.

The latter authors justify the use of dedicated instrumentation to achieve proper component positioning, to avoid eccentric reaming of the acetabulum and varus alignment of stems, to reduce stresses on the soft tissues and to avoid skin damage like abrasion. The creation of the mobile window allows the surgeon to perform hip replacement with the smallest incision that makes the introduction of the prosthetic components possible.

From this point of view, modified instrumentation is useful for the preservation of soft tissues. Flanged Hohmanns, dedicated reamers and broach handles adapt easier than standard instrumentation in the narrow surgical routes of minimally invasive approaches. In our clinical practice, we have experienced on lateral mini-approach and postero-lateral mini-approach. We use routinely flanged retractors which can be useful for better visualization of the acetabulum because with single instrument it is possible to retract soft tissues and femoral metaphysis. Angled handles greatly assist in the reaming of the acetabulum and angled inserter became necessary if we are going to implant a neck retaining stem. In fact they allow the surgeon to avoid eccentric reaming of the acetabulum and achieve proper orientation of the shell.

The evolution of minimally invasive surgery is TSS that involves the use of minimally invasive approaches and conservative prostheses. The effort is not focused on the skin incision length but mainly on reducing trauma of soft tissue and skeletal segments. Dedicated instrumentation adapted to the operative technique and to each prosthetic model is useful to reduce tissue damage and to achieve proper component positioning in total hip arthroplasty.

Conflict of interest statement The authors declare that they have no conflict of interest related to the publication of this manuscript.

\section{References}

1. Pipino F (2006) Tissue-sparing surgery (T.S.S.) in hip and knee arthroplasty. J Orthop Traumatol 7:33-35

2. Höwell JR, Masri BA, Duncan CP (2004) Minimally invasive versus standard incision anterolateral hip replacement: a comparative study. Orthop Clin N Am 35:153-162

3. D'Imporzano M, Pierannunzii L (2006) Minimally invasive total hip replacement. J Orthop Traumatol 7:42-50

4. Hartzband MA (2004) Postero-lateral minimal incision for total hip replacement: technique and early results. Orthop Clin N Am 35:119-129

5. Berger RA (2004) Mini incision total hip replacement using anterolateral approach. Orthop Clin N Am 35:143-151

6. Pipino F (2004) CFP prosthetic stem in mini-invasive total hip arthroplasty. J Orthop Traumatol 4:165-171

7. Ogonda L, Wilson R, Archbold P et al (2005) A minimal incision technique in total hip arthroplasty does not improve early postoperative outcomes. J Bone Joint Surg 87-A:701-710 
8. Woolson S, Mow C, Syquia J et al (2004) Comparison of primary total hip replacements performed with a standard incision or a mini incision. J Bone Joint Surg Am 86:1353-1358

9. O'Brien D (2005) The mini-incision direct lateral approach in primary total hip arthroplasty. Clin Orthop Relat Res 441:99-103
10. Chimento GF, Pavone V, Sharrock NE et al (2005) Minimally invasive total hip arthroplasty: a prospective randomized study. J Arthroplasty 20:139-144

11. Dorr LD (2003) Single-incision minimally invasive total hip arthroplasty. J Bone Joint Surg Am 85:2236-2238 